\title{
АНАЛИЗ ВЛИЯНИЯ УВЕЛИЧЕНИЯ МОЩНОСТИ ИСПОЛЬЗУЕМОГО АНСАМБЛЯ ТАЙМЕРНЫХ СИГНАЛЬНЫХ КОНСТРУКЦИЙ ПРИ ПОСТОЯННОЙ ДЛИТЕЛЬНОСТИ КОДОВЫХ КОНСТРУКЦИЙ НА СКОРОСТЬ ПЕРЕДАЧИ ИНФОРМАЦИИ В ЦИФРОВЫХ СИСТЕМАХ СВЯЗИ
}

\author{
Захарченко Н.В. ${ }^{1}$, Гаджиев М.М. ${ }^{2}$, Иванова Л.В. ${ }^{3}$, Сулима Ю.Ю. ${ }^{4}$, Шпак Д.М. ${ }^{5}$, Гордийчук В.В. ${ }^{6}$ \\ $1,2,5,6$ - Одесская национальная академия связи им. А.С. Попова, Одесса, Украина \\ 3,4 - Одесский технический колледж Одесской национальной академии пищевых технологий, Одесса, Украина \\ ORCID: ${ }^{2}$ http://orcid.org/0000-0001-7280-3863, ${ }^{3}$ http://orcid.org/0000-0003-1738-7697, ${ }^{4}$ http://orcid.org/0000-0003-3986- \\ 7296, ${ }^{5}$ http://orcid.org/0000-0002-8092-8276

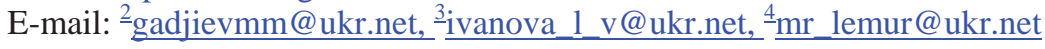

Copyright ( 2018 by author and the journal “Automation technologies and business - processes. This work is licensed under the Creative Commons Attribution International License (CC BY). http://creativecommons.org/licanses/by/4.0

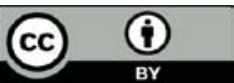

DOI:

Аннотация: В статье представлены результаты исследования влияния увеличения мощности используемого ансамбля таймерных сигнальных конструкций при постоянной длительности кодовых конструкций на скорость передачи информации в ичифровых системах связи. Обоснована целесообразность увеличения мощности используемого ансамбля таймерных сигнальных конструкиий, путем изменения числа информационных отрезков в кодовых конструкциях в системах передачи информации, использующци ичифровую связь. Проведен анализ методов передачи информащии в цүифровой связи при использовании позиционного способа кодирования с избыточньми кодами. Обоснована нецелесообразность использования позиционного кодирования при передаче больших объемов информации. Представлен метод кодирования сигнала на основе таймерных сигнальных конструкций с использованием отдельных временных отрезков сигнала, не менее найквистового интервала для каждой сигнальной конструкиии. Оцениваются временные параметры кодовых слов таймерных сигнальных конструкций: энтропия и информационная емкость найквистового элемента при кодовом уплотнении канала. Представлены результатьы исследования выбора эффективной длины кодового слова в методе кодирования сигнала с использованием таймерных сигнальных конструкций для увеличения скорости передачи информации в ичфровых системах связи. Приведены результаты исследования влияния параметров таймерных сигнальных конструкиий на скорость передачи информации, которые показали эффективность использования метода кодирования сигнала с использованием таймерных сигнальных конструкциий по сравнению с методом позиционного кодирования с избыточными кодами за счет увеличения информационной емкости найквистового элемента.

Annotation: The article presents the results of the study of the effect of increasing the power of the used ensemble of timer signal constructions with a constant duration of code structures on the speed of information transfer in digital communication systems. The expediency of increasing the power of the used ensemble of timer signal constructions is justified by changing the number of information segments in code structures in information transfer systems using digital communication. The analysis of methods of information transfer in digital communication using the positional coding method with redundant codes has been carried out. The inexpediency of using positional coding in the transfer of large amounts of information is substantiated. A method of coding a signal based on timer signal constructions using separate time intervals of the signal, not less than the Nyquist interval for each signal structure is presented. The time parameters of the code words of the timer signal constructions are estimated: entropy and information capacity of the Nyquist element during the channel code compression. The article presents the results of the study on the choice of the effective length of a code word in a signal coding method using timer 
signal structures to increase the speed of information transfer in digital communication systems. The results of the study of the influence of the parameters of the timer signal structures on the information transfer rate are shown, which showed the efficiency of using the signal coding method using timer signal structures compared to the positional coding method with redundant codes due to an increase in the information capacity of the Nyquist element.

Ключевые слова: методы передачи информации, цифровая связь, позиционные коды, кодовое слово, кодовые конструкции, таймерные сигнальные конструкции, найквистовый элемент, найквистовый интервал.

Keywords: methods of information transfer, digital communication, position codes, code word, code constructions, timer signal constructions, Nyquist element, Nyquist interval.

\section{1. Информационные параметры позиционных кодов}

1) Методы передачи информации в цифровой связи [1] обеспечивают передачу сообщений от дискретных источников, имеющих счётное (конечное) число состояний $N_{m}$.

$$
N_{m}=\sum_{i=0}^{n-1} d_{i} a^{i} \quad\left(i \in 0 \div i_{g} d>1\right)
$$

где $d_{i}$ - значение разрядных коэффициентов, которые не превышают значения;

$a$ - число различных значений информационного параметра в канале.

Заметим, что в реальной двоичной системе с позиционным кодированием $a=2$.

Максимальное число размеченных комбинаций при соблюдении условий для $d_{i}$ и $a$ равно [3].

$$
N_{m}=a^{n}
$$

где $\mathrm{n}$ - число элементов в кодовом слове.

2) Количество информации в позиционных кодовых словах (Н) определяется числом информационных элементов в них. Если код простой [3], т.е. используются все $N_{m}$ кодовых слов, то

$$
H=\log _{2} N_{m}=\log _{2} a^{n}=n \log _{2} a
$$

Из выражения (1) следует, что если $a>2$, то при позиционном кодировании в каждом элементе содержится более одного бита информации. Существующие системы связи используют избыточные коды, в которых каждая комбинация содержит m элементов информационных и r элементов дополнительных (избыточных) [2].

$$
n=m+r
$$

Например, в коде с четным числом единиц информационными являются только (n-1) элементов, а в 9-элементном коде Хэмминга содержится 5 информационных элементов и 4 проверочных [3], следовательно, энтропия каждого кодового слова $\mathrm{H}=5$, а информационная емкость одного элемента $I_{H}=\frac{\mathbf{5}}{9}=0,5555 \ldots$

3) Следует заметить, что каждое кодовое слово при позиционном кодировании передается в канал коэффициентами $x_{i}$, длительность сигнала каждого из которых равна длительности элемента Найквиста [4].

где: F- полоса спектра сигнала.

$$
t_{0}=\frac{1}{\Delta F}
$$

В заключении этого раздела оценим возможности передачи 2-х символьных ансамблей текста русского языка при позиционном кодировании. Так для передачи 32 символов русского языка необходимо согласно (1) 5 элементов кода $\left(\log _{2} 32=5\right)$, а для передачи двух символьных ансамблей, $\left(N_{p}=32 \times 32=1024\right)$ необходимо $\log _{2} 1024=10$ элементов в кодовом слове (аналогично, для передачи 3-х символьных ансамблей длина кодовых слов будет, равной $3 \log _{2} 32=15$ элементов).

Таким образом можно сделать вывод, что при передаче одним кодовым словом информации 0-Z символов русского языка, существует линейная зависимость количества элементов в групповой комбинации от кратности Z, символьных ансамблей.

4) Следует заметить, что при различных методах, обеспечивающих формирование (32 $\left.{ }^{z}\right)$ различных комбинаций общее число элементов $\mathrm{n}$ в кодовом слове равно

$n_{\Sigma}=Z \log _{2} 32$

Например, реализацией будут $N_{p 1}=23$ и $N_{p 2}=27$. Для формирования 1024 кодовых слов можно воспользоваться одним кодовым словом с числом элементов $n_{1}=10$, или двумя кодовыми словами по 5 элементов $n_{1}=n_{2}=5$, или двумя кодовыми словами при $n_{1}=2$ и $n_{2}=8$ с подмножествами чисел реализаций $N_{p 1}=2^{2}$ и $N_{p 8}=2^{8}$, что обеспечит общее число реализаций $(4 * 256=1024)$. При $n_{1}=3$ и $n_{2}=7$ подмножества будут $2^{3}=8 ; \quad 2^{7}=128$; $\left(n_{\Sigma}=3+7=10\right)$ с тем же числом реализаций $(8 * 128=1024)$.

К недостаткам позиционного кодирования следует отнести:

1. Расстояние между соседними моментами модуляции кратно длительности найквистового элемента, что ограничивает мощность реализуемого множества на интервале $n$ элементов; 
2. Элементность кодового слова $n$ определяется числом различных состояний кодируемого источника $N_{K}$ [4]

$n=E^{+} \log _{2} N_{K}$

где $E^{+}$- символ “целого большего числа $\log _{2} N_{k}$ ”, из [1] и [2] следует, что максимальное число $N$ при двоичных сигналах не превышает $2^{n}$.

5) В корректирующих (избыточных) кодах граничное число дополнительных $r$ элементов определяется пределом Варшамова - Гильберта [1]

где $d_{0}$ - требуемое кодовое расстояние.

$$
2^{n-m}=2^{r} \geq \sum_{i=0}^{d_{o=2}} c_{n}^{i}-1
$$

В таблице 1 приведены значения чисел избыточных элементов $r$, при $m \in 1 \div 15$ при $d_{1}=3$ и $d_{2}=5$

Таблица 1 - Число проверочных элементов при $d \in$ const

\begin{tabular}{|c|c|c|c|c|c|c|c|c|c|c|}
\hline$d_{0} / m$ & 1 & 2 & 3 & 4 & 5 & 6 & 12 & 13 & 14 & 15 \\
\hline 3 & 2 & 3 & 3 & 3 & - & 4 & 5 & 5 & 5 & 5 \\
\hline 5 & 4 & 7 & 8 & 8 & 9 & 9 & 14 & 15 & 15 & 15 \\
\hline
\end{tabular}

Как следует из таблицы 1 при кодовом расстоянии $d=5$ и $m \geq 1$ интервалов реализаций, число избыточных элементов при позиционном кодировании большого числа информации значительно возрастает, что делает такой код неэффективным.

\section{2. Таймерные сигнальные конструкции}

В отличие от позиционного способа кодирования, когда информация о передаваемом разряде определяется видом сигнала на единичном (найквистовом) интервале, в таймерных сигнальных конструкциях (ТСК) информация “заложена" в продолжительностях (длинах) i отдельных временных отрезков сигнала $\tau_{c i}$ на интервале каждой сигнальной конструкции $T_{\text {ск }}$. С целью уменьшения межсимвольных искажений продолжительность каждого из отрезков сигнала в сигнальной конструкции не менее найквистового интервала [2].

где $z \Delta \in 0 \div z_{0}-$ целые числа.

$$
\tau_{c i}=t_{0}+z \Delta
$$

Временной отрезок показывает часть единичного элемента $t_{0}>\Delta=\frac{t_{0}}{s}$ и определяется параметрами помех в канале и допустимой вероятностью ложного приема сигнальной конструкции $\left(s \in 2,4, \ldots, s_{0}\right)$.

Первое слагаемое выражения (2) обеспечивает установление переходного процесса на выходе канала при передаче всех $i$ отрезков каждой сигнальной конструкции, а второе $(z \Delta)$ несет информацию о кодовом слове.

Авторами таймерных сигналов [5], определена мощность (число реализации ТСК $\left.\left(N_{p}\right)\right)$ на интервале т найквистовых элементов:

$$
N_{p}=C_{m s-i(s-1)}^{i}
$$

После разложения выражения (3) получаем:

$$
N_{p}=\frac{(m s-i(s-1)) !}{i !(m s-i s) !}
$$

В таблице 2 приведены мощности $\left(N_{p}\right)$ ТСК при $s=7$ для $m \in 4 \div 8$ для $i \in 1 \div 7$

Из таблицы 2 следует сделать вывод, что число реализаций сигнальных конструкций увеличивается как при увеличении интервала реализаций $m$ при $i=$ const, так и при увеличении отдельных временных $i$ интервалов при $m=$ const .

Для выбора эффективной длины кодового слова проведём анализ изменения энтропии $(H)$ и информационной емкости найквистового элемента $\left(I_{H}\right)$ [3]. 
Таблица 2 - Мощности $N_{p}$ таймерных сигнальных конструкций

\begin{tabular}{|c|c|c|c|c|c|}
\hline $\mathrm{i}$ & $\mathrm{m}=4$ & $\mathrm{~m}=5$ & $\mathrm{~m}=6$ & $\mathrm{~m}=7$ & $\mathrm{~m}=8$ \\
\hline 1 & 22 & 29 & 36 & 636 & 946 \\
\hline 2 & 120 & 253 & 435 & 2925 & 5456 \\
\hline 3 & 120 & 680 & 1330 & 12650 & 35960 \\
\hline 4 & 1 & 330 & 3060 & 11628 & 65780 \\
\hline 5 & - & 1 & 792 & 1716 & 38760 \\
\hline 6 & - & - & 1 & 1 & 23450 \\
\hline 7 & - & - & - & - & 1 \\
\hline 8 & 263 & 1293 & 5654 & 29629 & 131643 \\
\hline$\Sigma$ & & - & - & & \\
\hline
\end{tabular}

При этом, учитывая работы [2], [3] определим значения вероятности появления отдельных событий $P_{i}$, а через значения последнего оценим $H$ и $I_{H}$.

$$
P_{i}=\frac{1}{N_{p}} ; H=\log _{2} N_{p} ; I_{H}=\frac{\log _{2} N_{p}}{m}
$$

В таблице 3 приведены значения информационной емкости найквистового элемента для $m \in 4 \div 10$ при s $\in 2 \div 10$ при $i=4$.

Таблица 3 - Информационная емкость найквистового элемента

\begin{tabular}{|c|c|c|c|c|c|c|c|}
\hline$s \backslash m$ & 4 & 5 & 6 & 7 & 8 & 9 & 10 \\
\hline 2 & 0 & 0,781378 & 1,021547 & 1,102035 & 1,118911 & 1,10747 & 1,082972 \\
\hline 3 & 0 & 1,025857 & 1,285708 & 1,354543 & 1,353715 & 1,324484 & 1,283664 \\
\hline 4 & 0 & 1,225857 & 1,491881 & 1,547103 & 1,530285 & 1,486146 & 1,432158 \\
\hline 5 & 0 & 1,395456 & 1,661204 & 1,702908 & 1,671914 & 1,615078 & 1,550109 \\
\hline 6 & 0 & 1,542849 & 1,804954 & 1,833806 & 1,790197 & 1,722343 & 1,647975 \\
\hline 7 & 0 & 1,673264 & 1,929886 & 1,946693 & 1,891763 & 1,814194 & 1,731615 \\
\hline 8 & 0 & 1,790257 & 2,04038 & 2,045939 & 1,980763 & 1,894511 & 1,804647 \\
\hline 9 & 0 & 1,89636 & 2,13944 & 2,134494 & 2,059969 & 1,965873 & 1,869462 \\
\hline 10 & 0 & 1,923445 & 2,229218 & 2,214442 & 2,131325 & 2,030079 & 1,927725 \\
\hline
\end{tabular}

Из таблицы 3 следует:

1) с увеличением $m$ при $s=$ const информационная емкость найквистовогоэлемента увеличивается до $m_{\text {мак }}$ (после $m_{\text {мак }}-$ уменьшается), с увеличением $s$ при $m=$ const и $i=$ const увеличивается.

В таблице 4 приведены значения $I_{H}$ при значениях $\mathrm{m} \in 4 \div 10$ и изменяемом числе информационных отрезков $i \in 1 \div 6, s=7$ 

http://www.atbp.onaft.edu.ua/

Таблица 4 - Информационная емкость найквистового элемента

\begin{tabular}{|c|c|c|c|c|c|c|c|}
\hline $\mathrm{i} \backslash \mathrm{m}$ & 4 & 5 & 6 & 7 & 8 & 9 & 10 \\
\hline 1 & 1,114 & 0,971 & 0,86 & 0,775 & 0,705 & 0,648 & 0,6 \\
\hline 2 & 1,726 & 1,596 & 1,46 & 1,339 & 1,235 & 1,146 & 1,069 \\
\hline 3 & 1,727 & 1,882 & 1,831 & 1,733 & 1,633 & 1,533 & 1,443 \\
\hline 4 & 0 & 1,673 & 1,929 & 1,946 & 1,891 & 1,814 & 1,731 \\
\hline 5 & - & 0 & 1,604 & 1,929 & 2 & 1,984 & 1,932 \\
\hline 6 & - & - & 0 & 1,534 & 1,905 & 2,019 & 2,035 \\
\hline
\end{tabular}

Анализ данных таблицы 4 показывает:

1) При каждом значении $i$ с ростом $\mathrm{m}, I_{H}$ вначале увеличивается, после достижения $m_{\text {мак }}-$ уменьшается:

$$
\begin{array}{ll}
m_{\max }(i=1)=4 & (1,727) \\
m_{\operatorname{Max}}(i=2)=5 & (1,882) \\
m_{\operatorname{Max}}(i=3)=6 \quad(1,929) \\
m_{\operatorname{Max}}(i=4)=7 \quad(1,946) \\
m_{\operatorname{Max}}(i=5)=8 \quad(2,00) \\
m_{\max }(i=6)=10 \quad(2,035)
\end{array}
$$

2) Для увеличения информационной емкости найквистового элемента можно суммировать информационные емкости при $m=$ const с различным числом информационных отрезков.

\section{Заключение}

Исследования влияния параметров таймерных сигнальных конструкций на скорость передачи информации показало, что изменяя число информационных отрезков $i$ в кодовых словах таймерных кодов при постоянном значении интервала реализаций $m=$ const можно увеличивать скорость передачи информации.

\section{Список использованных источников}

[1] Захарченко Н.В. Системи передачі даних. Том1. Ефективність блокового кодування: Підручник. / Н.В. Захарченко, В.Й. Кільдишев, О.М. Мартинова // ОНАЗ ім. О.С. Попова. - Одеса:2014 - 486с.

[2] Захарченко Н.В. Информационные параметры таймерных сигнальных конструкций при кодовом уплотнении канала / Н.В. Захарченко, С.М. Горохов, А.В. Кочетков, В.М Горицкий // Наукові праці ОНАЗ ім. О.С. Попова. Одесса:2016 - №2 - С.25-29.

[3] Захарченко Н.В. Сравнение позиционного и таймерного кодирования / Н.В. Захарченко, С.М. Горохов, А.В. Кочетков, В.В. Гордийчук // Збірник наукових праць віськового інституту телекомунікацій та інформатизації. Одесса:2016 - №2 - С.41-46.

[4] Зюко А.Г. Помехоустойчивость и эффективность систем передачи информации: Учебник./ А.Г. Зюко, А.Н. Филько, В.Л. Банкет, П.В. Иващенко // Радиосвязь - Москва:1985 - 304c.

[5] Захарченко Н.В. Информационные параметры таймерных сигналов / Н.В Захарченко, С.М. Горохов, А.В. Кочетков // Наукові праці ОНАЗ ім. О.С. Попова. - Одесса:2016 - №7 - С.24-28.

\section{References}

[1] N. V. Zakharchenko, V.Y. Kildyshev, O. M. Martynova, and V. Y. Kildyshev, Efektyvnist blokovoho koduvannia, vol. 1. Odessa: ONAZ im. O.S. Popova, 2014.

[2] N.V. Zakharchenko, S.M. Horokhov, A.V. Kochetkov, and V.M Horytskyi, Ynformatsionnyie parametryi taymernyih signalnyih konstruktsiy pry kodovom uplotnenyy kanala, Naukovi pratsi. Odessa:ONAZ im. O.S. Popova. - №2 pp.25-29., 2016.

[3] N.V. Zakharchenko, S.M. Horokhov, A.V. Kochetkov, and V.V. Hordyichuk, Sravnenye pozytsyonnoho y taimernoho kodyrovanyia, Zbirnyk naukovykh prats viskovoho instytutu telekomunikatsii ta informatyzatsii. - Odessa. - №2 pp.41-46., 2016.

[4] A.H. Ziuko, A.N. Fylko, V.L. Banket, P.V. Yvashchenko, Pomekhoustoichyvost y effektyvnost system peredachy ynformatsyy: Uchebnyk. Radyosviaz - Moskva. - 304p., 1985.

[5] N.V Zakharchenko, S.M. Horokhov, A.V. Kochetkov, Ynformatsionnyie parametryi taymernyih syhnalov. Naukovi pratsi, Odessa: ONAZ im. O.S. Popova. - №7 - pp.24-28., 2016. 